\title{
Noise Detection in Composite Signals by Analysing Signal Waveforms
}

\author{
Sarika Malhotra \\ Member IEEE \\ Associate Professor, \\ RKGIT , Ghaziabad, India.
}

\author{
R.K Singh \\ Professor ECE Dept, \\ Kumaon Engg College, \\ Dwarahat, India
}

\begin{abstract}
The Composite Signals are the combination of multi utility signals, compressed signals and coded signals to carry the information from source to destination. This information may be a digital signal, video information or data signal required as an input for the destination module. Presently the Composite Signals are basic components of any signal transmission system. In this paper we present an approach to detect the noise in composite signals by analysing signal waveforms.
\end{abstract}

\section{General Terms}

Composite Signal Wave forms, Noise Detection, Composite Signal Amplitude.

\section{Keywords}

Composite Signal, Signal decoding, Composite Signal Generation, Luminance Level, Signal Transmission.

\section{INTRODUCTION}

The Composite Signals are the signals which comprises of small signal components fused to generate a desired response. The outcome of the Composite Signals may results into video image of a remote data or the binary information, representing the output of a measurement system. The Composite Signals are transmitted through various channels and mediums[5]. During signal propagation the noise/ interference signals are introduced into the Composite Signals. This unwanted signal also gets mixed with the original signal in such a way that the overall outcome of the system is corrupted[1].

The nature of noise has to be determined to recover the original signal. The biggest challenge is to identify the noise patterns in the signal patterns. The visualization of the Composite Signals is executed by utilizing a CRO with high sampling capability[3].

\section{COMPOSITE SIGNAL VS NON COMPOSITE SIGNAL}

Composite Signals comprises of combination of small and significant signal components where each component contains specific information. The small signal components may have very small in amplitude but very significant signal which can't be ignored. The data associated with a discrete component can be altered. Where as a Non Composite Signal comprises of fixed amplitude signal information. If the amplitude of the signal is altered, the overall information value gets affected. Figure No.1 shows the waveforms of composite vs. Non Composite Signal.

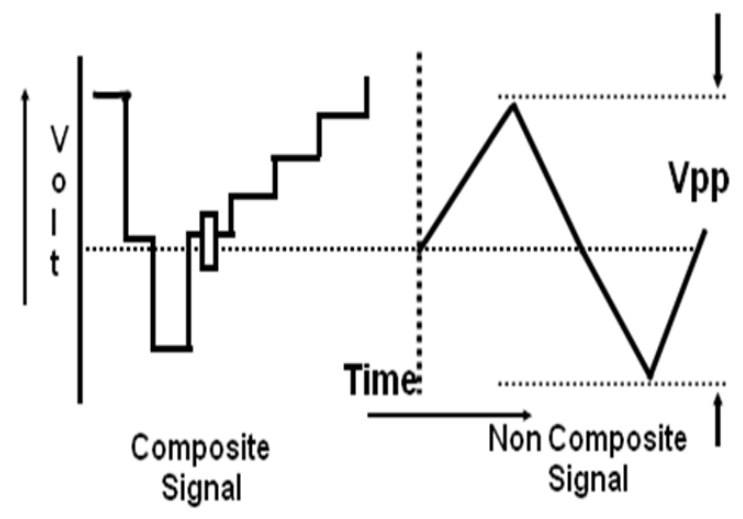

Fig No.1: Composite and Non Composite Signal

The Composite Signal in Figure No. 1 is Grey Scale pattern's waveform. The signal contains information regarding luminance level, colour level, synchronisation pulses and step amplitude for a grey scale signal[8]. Each component of the signal can be altered to change the respective artefact of the signal. The Non Composite Signal in figure No.1 is a triangular waveform which has two signal values in the form of pulse width and pulse amplitude. The alteration in such signals can be performed by altering time or overall amplitude of the signal [7].

\section{NOISE DETECTION IN COMPOSITE SIGNAL}

\subsection{Detection of Noise}

The detection of noise in Composite Signals is difficult as the noise does not alter the overall parametric characteristics of the wave forms. The variation in the amplitude of the signal is negligible whereas the change in the attributes of the signal 
components is major. The change in the characteristics of the signal components changes the overall response of the Composite Signal.

\subsection{Composite Signal waveforms}

The outcome of composite signals are analysed by utilising a high sampling rate CRO. The high sampling rate enables us to capture the minute details of the signal components of the composite signal. The signal source is connected to the input of the CRO to analyse the pattern of the composite signal. The figure No. 2 shows the captured image of the Color Bar pattern, generated by a composite signal generator[4].

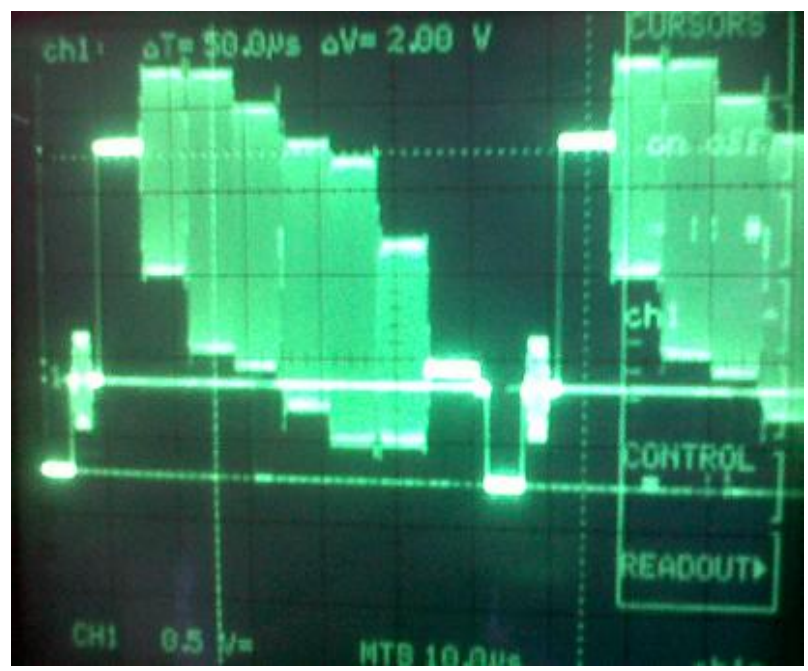

Fig No.2: Composite and Non Composite Signal

In The Figure No. 2 the waveform of Color Bar pattern is decoded with the help of a CRO[8]. The waveform clearly indicates the presence of

\section{a. Luminance Signal \\ b. Chrominance Signal \\ c. Sync signal \& Porches}

The Observed waveform on CRO is free from any interference or noise. All the component of the waveforms are clearly visible in a high sampling rate CRO. The selected sampling rate is $400 \mathrm{MS} / \mathrm{s}$.

\subsection{Distorted Composite Signals waveforms}

The Composite Signal is distorted with Step Grey Bar signal and transmitted through transmission channels. The waveform at the receiver end is analysed again with the help of CRO[5].

The figure No.3 shows the waveforms of distorted Composite Signal[6]. The waveform of the signal received is compared with the waveforms observed before transmission[7]. The end results obtained indicated that the signal waveforms received at the receiver end is overlapped by the signal of Step grey signal. The presence of the Step Grey signal deviates the standard waveforms from its original values to a new stated values[9]. These new values attained by the composite signal distort the original data/information[10].

The overlapped information produces undesirable effects on the final output monitor and thus the aim to send the information is lost[11]. By observing the waveforms on a
CRO the type and pattern of noise is easily detected[12]. Figure No.3 illustrates the Composite wave forms received at the receiver end [2]. The waveforms observed at the receiver end indicates that overall amplitude of the Composite Signal has not changed. It is also is also observed that the timing cycle of the signal also remains same. The change is observed in the allocation of signal components only.

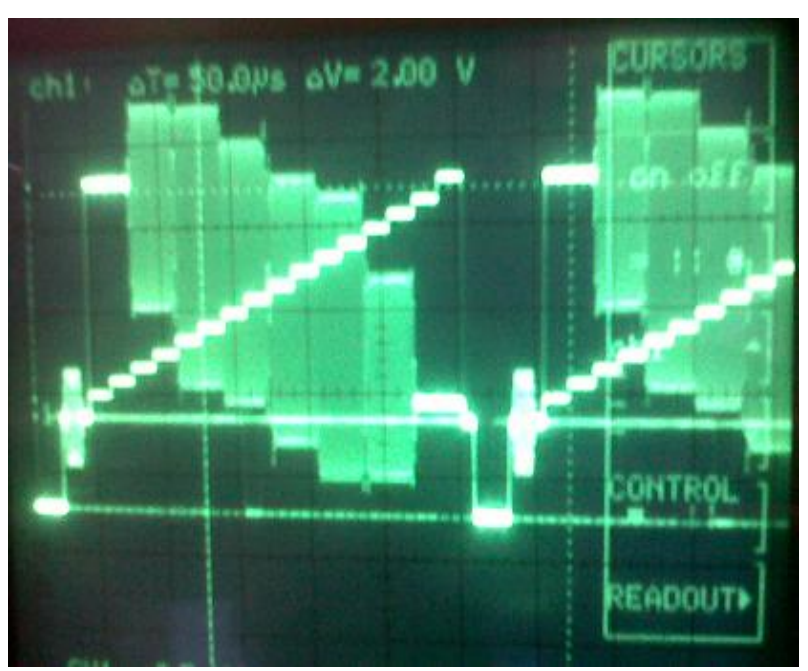

Fig No. 3. :Distorted Composite Signal

\section{RESULT}

The Noise in Composite Signal Waveforms is visualised on cathode ray oscilloscope. The pattern observed explains that the introduction of noise to the composite signal alters the signal components of the original waveform whereas the overall amplitude remains unchanged.

Table 1. Signal Components of Distorted Vs Non Distorted Composite Signals

\begin{tabular}{|c|c|c|c|}
\hline Waveform & $\begin{array}{c}\text { No. of } \\
\text { Steps }\end{array}$ & Video Level & $\begin{array}{c}\text { Chrominance } \\
\text { Level }\end{array}$ \\
\hline Original & 6 & $1.00 \mathrm{~V}$ & $0.300 \mathrm{mV}$ \\
\hline Distorted & $6+18$ & $1.00 \mathrm{~V}$ & $0.300 \mathrm{mV}$ \\
\hline
\end{tabular}

The table No. 1 indicates that the distortion in the composite signals is detected by comparing the distorted signal with respect to the Un distorted signal. The comparison of the two signals reveals the presence of unwanted signal

\section{CONCLUSION}

The Noise in Composite Signal waveforms is detected by observing the waveforms on a CRO. The effect of noise on the composite signal is determined by measuring the composite signal's amplitude and timing values. The pattern of noise and its effects on the transmitted Composite Signal is estimated to remove Noise from the composite signal..

\section{ACKNOWLEDGMENTS}

Our thanks to M/s Global Calibration laboratory for providing the practical setup for the analysis of composite signals. 


\section{REFERENCES}

[1] $\mathrm{Hu}$ Chen, Mingzhe Sun, and Eckehard Steinbach, "Compression of Bayer-Pattern Video Sequences Using AdjustedChroma Subsampling" IEEE Transaction on Circuits and systems for video Technology, Vol. 19, NO. 12, DEC 2009.

[2] Ji Won Lee, Hyun-Seung Lee, Rae-Hong Park, and Sunghee Kim, Member, "Reduction of Dot Crawl and Rainbow Artifacts in the NTSC Video" IEEE Transactions on Consumer Electronics, Vol. 53, No. 2, MAY 2007.

[3] A Punchihewa , D.G Bailey and R. M. Hodgson, "Objective Quality Assessment of Coded Images : The Development of New Quality Metrics," Proceedings of Internet, Telecommunication Conference, Adelaide, Australia, pp. 1-6, 2004.

[4] Tetsui Ryoichi " Signal generator of digital -TV generation LCD " Semicond FPD word proceeding, Vol 23, page 94-99, 2004

[5] Dr. Saupe \&Dr. Xiong Book on "“ Efficient Error Protection for image and video transmission over noisy channels "Univ. Konstanz \& Univ. A\&M Texas precceding May2004.

[6] Jaeheon Lee, Yeong-Hwa Kim, and Ji-Ho Nam "Adaptive Noise Reduction Algorithms based on
Statistical Hypotheses Tests" IEEE Transactions on Consumer Electronics, Vol. 54, No. 3, AUGUST 2008.

[7] Tanaka Y, “Video Signal Noise Characteristics with regard to scanning Method". IEEE Proceeding, Vol 40 Issue 2, PP 68 - 74, Aug 2006.

[8] Van Roermund, Snijder R.j Philips Res. Lab “ A general Purpose programmable video signal Processor "IEEE proc. Vol. 35, Issue 3, PP 249 - 258, August1989.

[9] A Peiravi \& S Toosizadeh, "Automatic Adjustment of Television sets using a unacalibrated camera with a Novel Fuzzy test Pattern and an Adaptive Algorithm ". Journal of Applied Sciences , Ferdowsi Univ, Mashhad, Iran Asian Network for scientific Information, Vol 9(1), PP 49-58, 2009

[10] Tsuyoshi Harada , Yoshie Oritake on "Video signal defect compensation system ", US Patent No 4315276 ,US Classification 358/8 ; 360/38 , Feb 1982.

[11] J Carl hooper, Dr. Sunnyvale, sydney, "Noise Reduction System for Video Signals" US Patent No. PP 1 to 15, Feb 1986.

[12] A. Punchihewa, D. G. Bailey and R. M. Hodgson, "A Survey of Coded Image and Video Quality Assessment," Proceedings of Image and Vision Computing New Zealand, Palmerston North, New Zealand, pp. 326-331, 2003 